\title{
Improvement of thermostability and catalytic efficiency of glucoamylase from Talaromyces leycettanus JCM12802 via site-directed mutagenesis to enhance industrial saccharification applications
}

Lige Tong, Jie Zheng, Xiao Wang, Xiaolu Wang, Huoqing Huang, Haomeng Yang, Tao Tu, Yuan Wang,

Yingguo Bai, Bin Yao, Huiying Luo and Xing Qin*

\begin{abstract}
Background: Glucoamylase is an important industrial enzyme in the saccharification of starch into glucose. However, its poor thermostability and low catalytic efficiency limit its industrial saccharification applications. Therefore, improving these properties of glucoamylase is of great significance for saccharification in the starch industry.

Results: In this study, a novel glucoamylase-encoding gene TIGa15B from the thermophilic fungus Talaromyces leycettanus JCM12802 was cloned and expressed in Pichia pastoris. The optimal temperature and pH of recombinant T/Ga15B were $65^{\circ} \mathrm{C}$ and 4.5 , respectively. T/Ga15B exhibited excellent thermostability at $60{ }^{\circ} \mathrm{C}$. To further improve thermostability without losing catalytic efficiency, T/Ga15B-GA1 and T/Ga15B-GA2 were designed by introducing disulfide bonds and optimizing residual charge-charge interactions in a region distant from the catalytic center. Compared with T/Ga15B, mutants showed improved optimal temperature, melting temperature, specific activity, and catalytic efficiency. The mechanism underlying these improvements was elucidated through molecular dynamics simulation and dynamics cross-correlation matrices analysis. Besides, the performance of T/Ga15B-GA2 was the same as that of the commercial glucoamylase during saccharification.
\end{abstract}

Conclusions: We provide an effective strategy to simultaneously improve both thermostability and catalytic efficiency of glucoamylase. The excellent thermostability and high catalytic efficiency of T/Ga15B-GA2 make it a good candidate for industrial saccharification applications.

Keywords: Glucoamylase, Thermostability, Catalytic efficiency, Site-directed mutagenesis, Industrial application

\section{Background}

Glucoamylase (1,4- $\alpha$-D-glucan glucohydrolase, EC 3.2.1.3) is one of the most important and widely used industrial enzymes, exhibiting great application potential

*Correspondence: qinxing@caas.cn

State Key Laboratory of Animal Nutrition, Institute of Animal Sciences,

Chinese Academy of Agricultural Sciences, Beijing 100193, China in sugar, ethanol, bread, beer, textile, and pharmaceutical industries [1]. Glucoamylase could catalyze the hydrolysis of $\alpha-1,4$ and $\alpha-1,6$ glycosidic linkages in starch and related oligosaccharides from non-reducing ends to release $\beta$-D-glucose [2]. Currently, commercial glucoamylases are mainly obtained from filamentous fungi, such as Aspergillus niger, Rhizopus niveus, and $R$. delemar, displaying moderate thermostability and slow catalytic 
activities [3, 4]. However, the industrial conversion of starch to glucose consists of a liquefaction process at $95-105{ }^{\circ} \mathrm{C}$ for $2 \mathrm{~h}$ and a saccharification process at $60{ }^{\circ} \mathrm{C}$ for $48-72 \mathrm{~h}$. Switching from the liquefaction to the saccharification process requires additional specialized equipment for cooling to ensure the catalytic integrity of glucoamylase [5]. The poor thermostability of current glucoamylases results in great energy consumption and process inconvenience to undergo the saccharification process in starch industries.

Generally speaking, enzyme discovery and engineering are the major approaches for acquiring enzymes with higher thermostability and catalytic efficiency. Moreover, the search for thermostable glucoamylases derived from thermophilic microorganisms has attracted immense attention over recent years. So far, several thermostable glucoamylases have been identified from $A$. wentii, A. oryzae, A. flavus, Fomitopsis palustris, Chaetomium thermophilum, Rhizomucor pusillus, Thermoanaerobacter tengcongensis, and Sulfolobus solfataricus, all of which exhibit optimal temperature or thermostability above 60 ${ }^{\circ} \mathrm{C}$ [3, 6-12]. Nevertheless, further research is needed pertaining to the industrial aspect of the saccharification process. Reconstructing enzymes by protein engineering is another efficient way to obtain glucoamylases with high thermostability. Based on previous research, directed evolution and rational design, such as in the construction of disulfide bonds, result in significant thermostability improvement of glucoamylases. [13-16]. However, due to the activity-stability trade-off, an increase in enzyme thermostability is accompanied by a decrease in catalytic efficiency [17, 18].

Recently, it has been reported that increasing the overall rigidity of protein while minimizing influence on the active site could render improved thermostability either without affecting catalytic activity or ideally with positively promoting catalytic activity [19]. Moreover, many previous studies on phytase, $\beta$-glucanase, and xylanase thermostability engineering support this conclusion $[20$ 22]. Such studies indicate that selecting mutant sites in a region distant from the catalytic center may be feasible to design enzymes with increased thermostability and catalytic efficiency.

Talaromyces leycettanus JCM12802 is a typical thermophilic fungus with an optimum temperature for growth at $40{ }^{\circ} \mathrm{C}$, producing a wide range of thermostable glycoside hydrolases, such as cellulase, $\beta$-glucosidase, and mannanase [23-25]. In this study, a novel thermostable glucoamylase $T l G a 15 B$ from $T$. leycettanus JCM12802 was expressed and characterized. In order to further improve thermostability, TlGa15B mutants were rationally designed by introducing disulfide bonds and optimizing residual charge-charge interactions in a region distant from the catalytic center. Moreover, the application potential of the best mutant was evaluated by comparison with a commercial glucoamylase commonly used in the starch industry for saccharification.

\section{Results and discussion \\ Cloning and sequence analysis of $T / G a 15 B$}

In the present study, a novel glucoamylase-encoding gene $T l G a 15 B$ was identified in the genome of thermophilic fungus T. leycettanus JCM12802, and the corresponding cDNA sequences were successfully obtained from the fungus grown in PDB medium. The TlGa15B was interrupted by 4 introns, resulting in an open reading frame of $1842 \mathrm{bp}$. Deduced TlGa15B contained 614 amino acid residues and harbored a signal peptide of 20 residues based on predictions established by the SignalP 5.0 program [26]. The isoelectric point and calculated molecular weight of glucoamylase $T l G a 15 B$ were 4.76 and $63.3 \mathrm{kDa}$, respectively. $\mathrm{TlGa} 15 \mathrm{~B}$ shared the highest identity $(69.85 \%)$ with a previously reported glucoamylase derived from Rasamsonia emersonii [27]. The sequence and structure analysis showed that $T l \mathrm{Ga} 15 \mathrm{~B}$ was a typical glucoamylase, containing a catalytic domain of GH15 and a carbohydrate-binding domain of CBM20. Glu211, and Glu433 was predicted to be the catalytic residues that served as the proton donor and acceptor, respectively. Moreover, five highly conserved residues associated with substrate binding in the GH15 family were identified in the catalytic domain of $T l G a 15 \mathrm{~B}$, including Arg86, Asp87, Leu209, Trp210, Glu212, and Arg338 [28]. In addition, the modeled structure of $T l G a 15 B$ was constructed using the glucoamylase from Penicillium oxalicum (PDB: 6FHV, 58\% sequence identity and 94\% coverage) as template with the help of SWISS-MODEL. Meanwhile, the Ramachandran plot was used to evaluate the quality of the structural model. There were only two amino acid residues observed in disallowed region (Additional file 1), indicating that the modeled structure of $T l \mathrm{Ga} 15 \mathrm{~B}$ was credible.

\section{Expression and purification of recombinant TIGa15B}

Pichia pastoris, the microbial expression system most widely used for the large-scale production of commercial enzymes, was used as the recombinant expression host to express glucoamylase $T l \mathrm{Ga} 15 \mathrm{~B}$ [29]. The cDNA fragment coding for the mature $T l \mathrm{Ga} 15 \mathrm{~B}$ was inserted into PIC9 and successfully expressed in Pichia pastoris GS115. After methanol induction, the glucoamylase activity of the culture supernatant reached $5000 \mathrm{U} / \mathrm{L}$ at $48 \mathrm{~h}$. After purification by anion exchange, the recombinant $T l \mathrm{Ga} 15 \mathrm{~B}$ had an apparent molecular weight of $\sim 65 \mathrm{kDa}$ on the SDS-PAGE (Additional file 2), which was close to the theoretical molecular weight. 


\section{Biochemical characterization of purified recombinant TIGa15B}

The optimal $\mathrm{pH}$ of purified recombinant $T l \mathrm{Ga} 15 \mathrm{~B}$ was pH 4.5 (Fig. 1a). Similar pH optima for glucoamylase activities had been reported from $C$. thermophilum $\mathrm{pH}$ 4.5-5.0 [11], A. tritici pH 4.0-5.0 [30], and Bispora sp. 4.0 [31]. Meanwhile, TlGa15B exhibited high stability in $\mathrm{pH}$ values ranging from 2.0 to 12.0 (Fig. 1b). The residual hydrolytic activity of TlGa15B after $1 \mathrm{~h}$ of incubation was over $75 \%$ of the original activity prior to incubation. These results suggested that $T l \mathrm{Ga} 15 \mathrm{~B}$ was an acidic glucoamylase with broad-range $\mathrm{pH}$ stability.

As shown in Fig. 1c, the hydrolytic activity of purified recombinant $T l G a 15 B$ increased with increasing temperature, reaching a maximum at $65{ }^{\circ} \mathrm{C}$. The optimal temperature was the same as the most widely used glucoamylases from $A$. niger $[1,32]$. In terms of thermostability, TlGa15B from T. leycettanus JCM12802 exhibited excellent thermostability. $T l \mathrm{Ga} 15 \mathrm{~B}$ retained $79.5 \%, 54.8 \%$, and $36.7 \%$ of hydrolytic activity after $1 \mathrm{~h}$ of incubation at $55{ }^{\circ} \mathrm{C}, 60{ }^{\circ} \mathrm{C}$, and $65{ }^{\circ} \mathrm{C}$, respectively (Additional file 3 ). When the temperature increased to above $75{ }^{\circ} \mathrm{C}$, the thermostability of TlGa15B significantly decreased (Fig. 1d). In contrast, the thermostability of $T l \mathrm{Ga} 15 \mathrm{~B}$ is superior to that of most reported glucoamylase, which are stable at temperatures below $55{ }^{\circ} \mathrm{C}[1]$.

In addition, the kinetic parameters of purified $T l \mathrm{Ga} 15 \mathrm{~B}$ with respect to soluble starch were determined at 55 ${ }^{\circ} \mathrm{C}$. The $K_{\mathrm{m}}$ and $V_{\max }$ values of purified recombinant $T l \mathrm{Ga} 15 \mathrm{~B}$ were $0.77 \mathrm{mg} / \mathrm{mL}$ and $719.2 \mu \mathrm{mol} / \mathrm{min} / \mathrm{mg}$, respectively (Table 1 ). Meanwhile, the specific enzyme activities of purified recombinant $T l \mathrm{Ga} 15 \mathrm{~B}$ were also evaluated using various substrates, including soluble starch, amylose, glycogen, $\alpha$-cyclodextrin, $\beta$-cyclodextrin, and $\gamma$-cyclodextrin. The results revealed that $T l G a 15 B$ was active toward soluble starch, amylose, and glycogen, but not other substrates, such as $\alpha$-cyclodextrin, $\beta$-cyclodextrin, and $\gamma$-cyclodextrin. The specific activity toward glycogen was higher than toward soluble starch and amylose. The specific activities of the purified recombinant $T l G a 15 B$ to soluble starch, amylose, and glycogen were $496.2,221.1$, and $658.3 \mathrm{U} / \mathrm{mg}$, respectively.

\section{Selection of the mutagenesis sites in T/Ga15B}

It was reported that the construction of disulfide bonds and the optimization of residual charge-charge interactions were efficient strategies to improve the thermostability of industrial enzymes [33]. In this study,

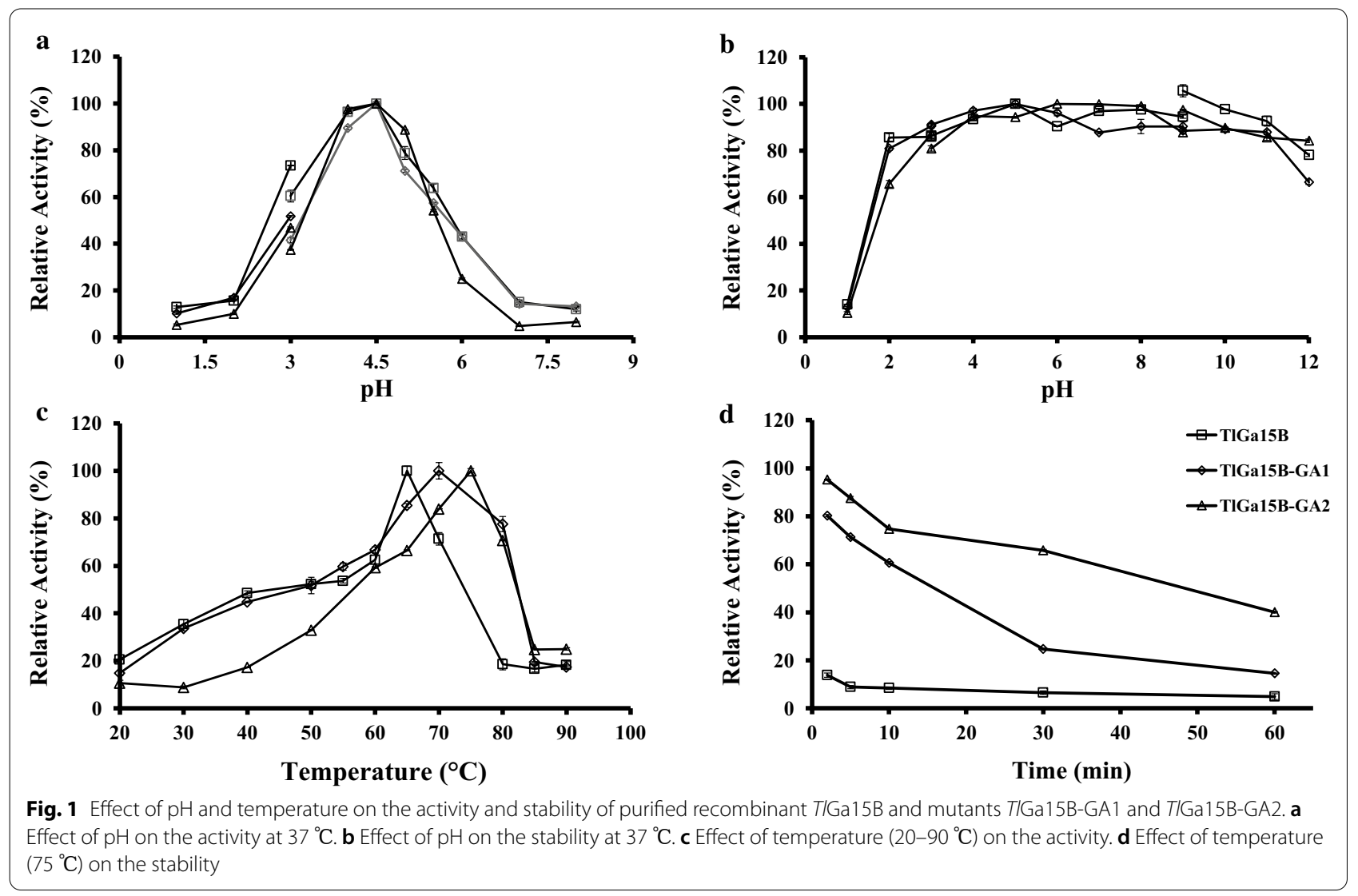


Table 1 The kinetic parameters of T/Ga15B and mutants on soluble starch at $55^{\circ} \mathrm{C}$

\begin{tabular}{lcccc}
\hline Enzyme & Specific activity $(\mathbf{U} / \mathbf{m g})$ & $\boldsymbol{K}_{\mathbf{m}}(\mathbf{m g} / \mathbf{m L})$ & $\boldsymbol{V}_{\max }(\boldsymbol{\mu m o l} / \mathbf{m i n} / \mathbf{m g})$ & $\boldsymbol{k}_{\text {cat }} / \boldsymbol{K}_{\mathbf{m}}(\mathbf{m L} / \mathbf{s} / \mathbf{m g})$ \\
\hline T/Ga15B & $496.2 \pm 1.3$ & $0.77 \pm 0.08$ & $719.2 \pm 19.1$ & 982.3 \\
T/Ga15B-GA1 & $805.8 \pm 5.7$ & $0.62 \pm 0.07$ & $900.9 \pm 23.2$ & 1529.0 \\
T/Ga15B-GA2 & $1054.5 \pm 2.2$ & $0.29 \pm 0.02$ & $1093.0 \pm 22.0$ & 3982.6 \\
\hline
\end{tabular}

these protein engineering approaches were applied to enhance the thermostability of $T l \mathrm{Ga} 15 \mathrm{~B}$. Based on multiple sequence alignment and structure analysis, S132, Y492, L548, and A562 were chosen as targets to construct two disulfide bonds. The mutant $T l$ Ga15B-GA1 (S132C-Y492C/L548C-A562C) was constructed via PCR-mediated site-directed mutagenesis. As shown in Additional file 4, two disulfide-linked peptides SNPSGGLCT/SASGPCA (132C/492C) and PLWYCIV/SAIPCSA $(548 \mathrm{C} / 562 \mathrm{C})$ were observed in the LC-MS/MS analysis, indicating that $T l G a 15 B$ mutants formed disulfide bonds in the target sites. In addition, the enzyme thermal stability system (ETSS) was used to calculate the total interaction energy $\left(E_{i j}\right)$ between charged amino acids of $T l G a 15 B$ [34]. 6 non-conserved amino acid residues (C94/E98/Q108/D289 /D296/ E473) in a region distant from the catalytic center but in the catalytic domain of glucoamylase were selected for further analysis by ETSS (Fig. 2; Additional file 5). On account of the ETSS analysis, Q108 (total $E_{i j}=27.3 \mathrm{~kJ} /$ mol) was mutated to a neutral amino acid alanine (total $E_{i j}=2.3 \mathrm{~kJ} / \mathrm{mol}$ ), exhibiting the most significant decline in the $E_{i j}$ value. Subsequently, the mutant $T l G a 15 B-$ GA2 (S132C-Y492C/L548C-A562C/Q108E) was generated via site-directed mutagenesis using $T l$ Ga15B-GA2 as the template.

\section{Comparison of the enzymatic properties of $T / G a 15 B$ and mutants}

As shown in Fig. 1a, b, there were no significant differences in $\mathrm{pH}$ optima and stability between $T l \mathrm{Ga} 15 \mathrm{~B}$ and its mutants $T l \mathrm{Ga} 15 \mathrm{~B}-\mathrm{GA} 1$ and $T l \mathrm{Ga} 15 \mathrm{~B}-\mathrm{GA} 2$. By comparison, the optimal temperature and thermostability of mutants both significantly improved. The optimal temperatures of $T l G a 15 B-G A 1$ and $T l G a 15 B-G A 2$ were 70 ${ }^{\circ} \mathrm{C}$ and $75{ }^{\circ} \mathrm{C}$, which were $5{ }^{\circ} \mathrm{C}$ and $10{ }^{\circ} \mathrm{C}$ higher than that of $T l G a 15 B$, respectively (Fig. 1c). The TlGa15B incubated at $75{ }^{\circ} \mathrm{C}$ for 2 min only had $13.8 \%$ residual enzyme activity, while the mutant $T l$ Ga15B-GA1 still contained $60.6 \%$ and $24.6 \%$ of enzyme activity after incubation at $75{ }^{\circ} \mathrm{C}$ for $10 \mathrm{~min}$ and $30 \mathrm{~min}$, respectively. Furthermore, the mutant $T l \mathrm{Ga} 15 \mathrm{~B}-\mathrm{GA} 2$ was extremely stable at $75{ }^{\circ} \mathrm{C}$, and $40 \%$ of enzyme activity remained after $1 \mathrm{~h}$ of incubation (Fig. 1d). Meanwhile, the DSC results supported the conclusion that both $T l$ Ga15B-GA1 and $T l$ Ga15B-GA2 exhibited higher thermostability than TlGa15B (Fig. 3). Compared with the $T_{\mathrm{m}}$ value of $T l \mathrm{Ga} 15 \mathrm{~B}\left(72{ }^{\circ} \mathrm{C}\right)$, the $T_{\mathrm{m}}$ values of $T l \mathrm{Ga} 15 \mathrm{~B}-\mathrm{GA} 1$ and $T l \mathrm{Ga} 15 \mathrm{~B}-\mathrm{GA} 2$ showed a significant increase of $8{ }^{\circ} \mathrm{C}\left(80^{\circ} \mathrm{C}\right)$ and $9{ }^{\circ} \mathrm{C}\left(81{ }^{\circ} \mathrm{C}\right)$, respectively.

Remarkably, $\quad T l$ Ga15B-GA1 and TlGa15B-GA2 also exhibited higher catalytic activity and efficiency than $T l$ Ga15B (Table 1; Additional file 6). The specific
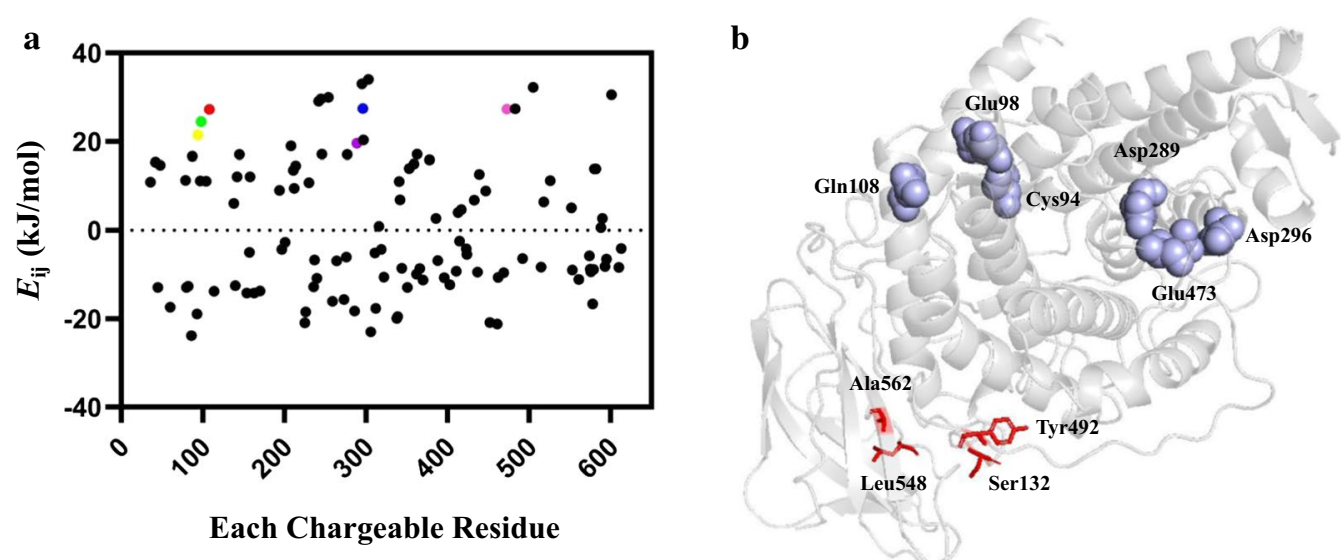

Fig. 2 Total interaction energy of each chargeable residue in T/Ga15B by ETSS analysis (a). The modeled structure of T/Ga15B (b). Six candidate mutated sites away from the catalytic center were indicated in blue balls. The sites for introduction of disulfide bonds were shown as red sticks 


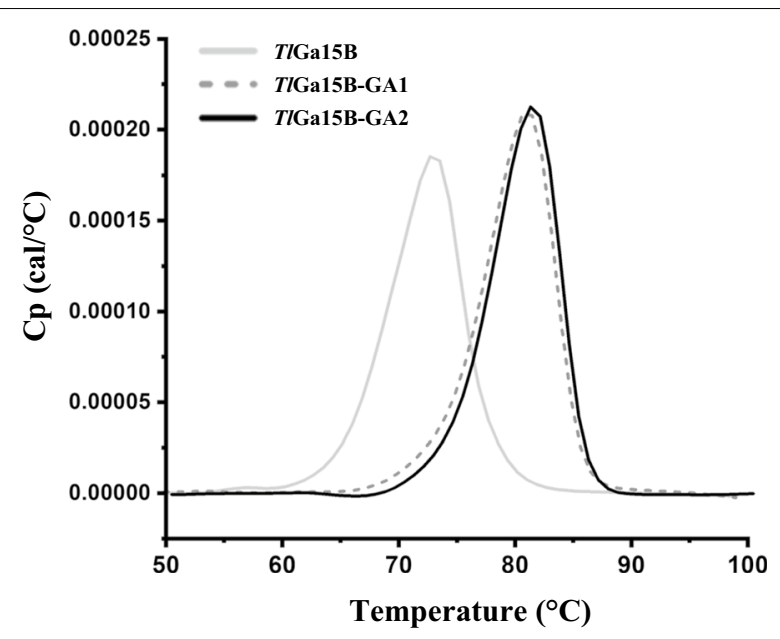

Fig. 3 Thermograms of T/Ga15B and mutants T/Ga15B-GA1 and T/Ga15B-GA2 detected using the DSC

activities of $T l \mathrm{Ga} 15 \mathrm{~B}-\mathrm{GA} 1$ and $T l \mathrm{Ga} 15 \mathrm{~B}-\mathrm{GA} 2$ were approximately 1.6 -fold and 2.2-fold higher than that of $T l \mathrm{Ga} 15 \mathrm{~B}$, respectively. In contrast to $T l \mathrm{Ga} 15 \mathrm{~B}$, the catalytic efficiencies of $T l G a 15 B-G A 1$ and $T l G a 15 B-G A 2$ increased by $55.7 \%$ and $305.4 \%$, respectively. Usually, thermostability improvement of enzymes is accompanied by a decrease in catalytic activity due to the activity-stability trade-off $[17,18]$. However, these results indicate that the activity-stability trade-off could be overcome by carefully selecting mutation sites distant from the catalytic center.

MD simulation and DCCM analysis of T/Ga15B and mutants In order to elucidate the mechanism underlying the thermostability of mutants $T l \mathrm{Ga} 15 \mathrm{~B}-\mathrm{GA} 1$ and $T l \mathrm{Ga} 15 \mathrm{~B}-$ GA2, MD simulations were carried out at $300 \mathrm{~K}$ for $20 \mathrm{~ns}$. The root mean square deviation (RMSD) was an important parameter to reflect the rigidity of the protein structure, which correlated with protein thermostability [35]. The RMSD values of $T l G a 15 B-G A 1$ and $T l G a 15 B-G A 2$ significantly decreased compared with that of $T l G a 15 B$, indicating that the overall structures of the mutants were more thermostable (Fig. 4a). Moreover, based on the root mean square fluctuation (RMSF) analysis, residues Q108, S132, Y492, L548, and A562 of TlGa15B were more flexible than the corresponding ones of the mutants TlGa15B-GA1 and TlGa15B-GA2 (Fig. 4b). Besides, the flexibilities of amino acid residues in the CBM domain of thermostable mutants were markedly decreased. The lower RMSF values of these amino acid residues rendered the CBM domain more stable, which might result in improved catalytic efficiency of $T l G a 15 B-G A 1$ and TlGa15B-GA2.
In addition, to understand the complex interaction between the selected mutated amino acid residues and the CBM domain, the DCCMs of $T l \mathrm{Ga} 15 \mathrm{~B}$ and $T l \mathrm{Ga} 15 \mathrm{~B}$ GA2 were calculated using the coordinates of the $\mathrm{C} \alpha$ atoms from the trajectories. As shown in Fig. 4c, d, the negative correlation between the amino acid residues (100-120) and the CBM domain (residues 490-613) was weakened in the mutant $T l$ Ga15B-GA2, thereby indicating a marked reduction in the movement of the negative correlation between them. These results were consistent with the conclusions of the RMSF analysis.

\section{Starch saccharification using T/Ga15B-GA2 in combination with pullulanase}

In order to evaluate the performance of industrial saccharification applications, the best mutant $T l \mathrm{Ga} 15 \mathrm{~B}-\mathrm{GA} 2$ was chosen for enzymatic saccharification of liquefied starch. As shown in Fig. 5, the maximum glucose productions for $T l G a 15 B-G A 2$ and the commercial glucoamylase GA-LD were obtained after $30 \mathrm{~h}$ of incubation at $60{ }^{\circ} \mathrm{C}$. The DX value (glucose content) for $T l \mathrm{Ga} 15 \mathrm{~B}-\mathrm{GA} 2$ and GA-LD reached $96.4 \%$ and $96.7 \%$, respectively, indicating no significant difference between $T l$ Ga15B-GA2 and the commercial glucoamylase GA-LD. Moreover, the DX value of $T l \mathrm{Ga} 15 \mathrm{~B}-\mathrm{GA} 2$ was in accordance with the industrial requirement for the production of glucose with a DX value above $96 \%$. These results suggested that $T l G a 15 B-G A 2$ had the same saccharification effect as industrial glucoamylases for the enzymatic saccharification process of starch.

\section{Conclusions}

TlGa15B, derived from thermophilic fungus T. leycettanus JCM12802, exhibited high optimal temperature and excellent thermostability above $60{ }^{\circ} \mathrm{C}$. By introducing disulfide bonds and optimizing residual charge-charge interactions in a region distant from the catalytic center, TlGa15B-GA1 and TlGa15B-GA2 showed improvements in optimal temperature, melting temperature, specific activity, and catalytic efficiency. The mechanism underlying the improved thermostability and catalytic efficiency of glucoamylase was elucidated by MD simulation and DCCM analysis. The performance of $T l \mathrm{Ga} 15 \mathrm{~B}-\mathrm{GA} 2$ was similar to that of the commercial glucoamylase during the saccharification process of starch. These properties of $T l G a 15 B-G A 2$ make it a promising candidate for industrial saccharification applications.

\section{Methods}

\section{Strain and substrates}

The thermophilic fungus T. leycettanus JCM12802 was purchased from the Japan Collection of Microorganisms RIKEN BioResource Center (Tsukuba, Japan) and 

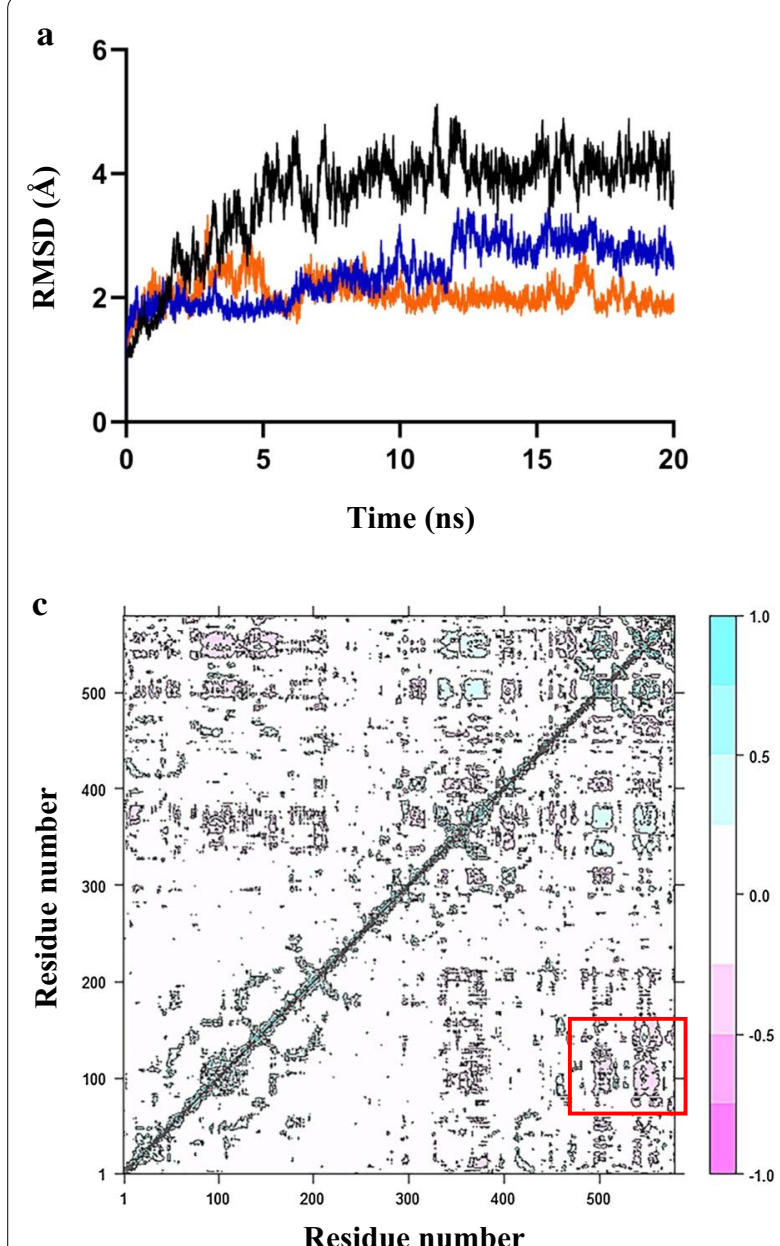
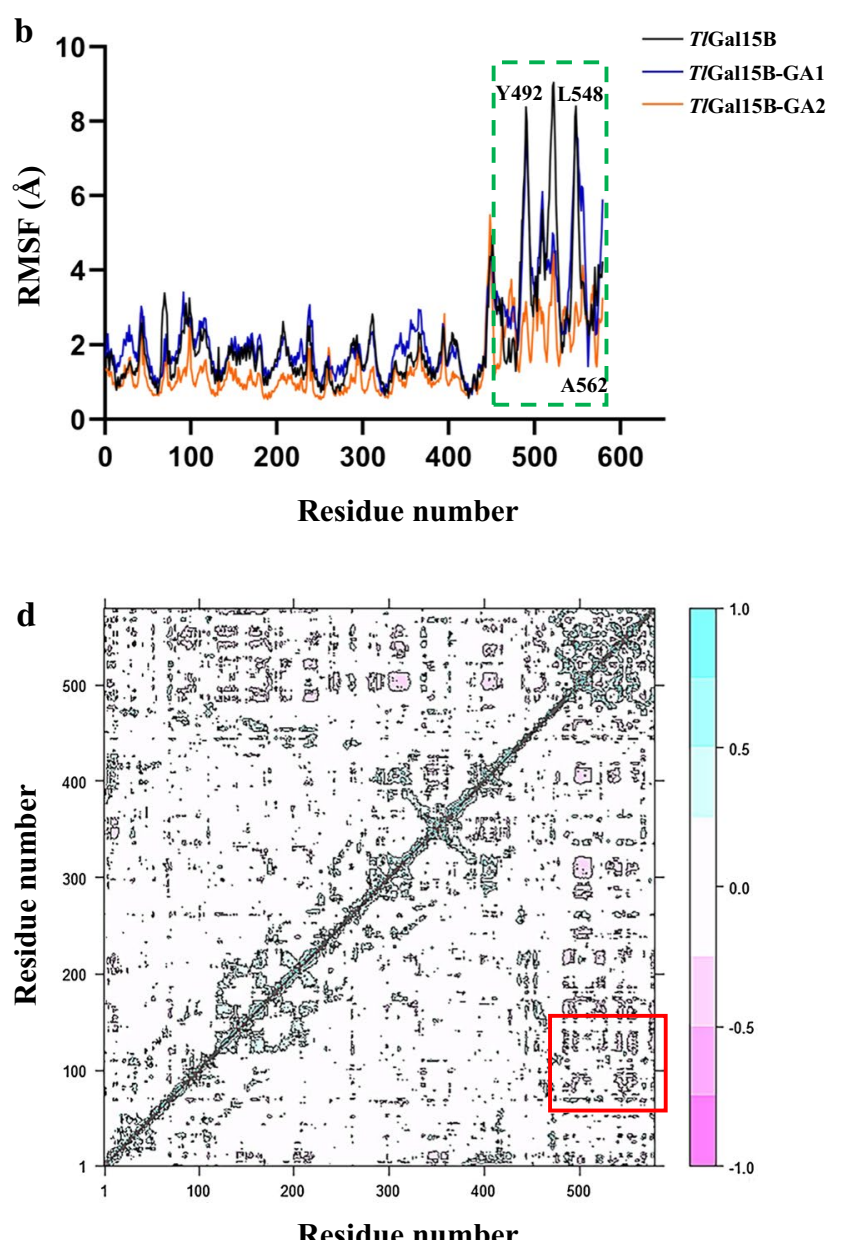

Fig. 4 MD simulation analysis of T/Ga15B and mutants T/Ga15B-GA1 and T/Ga15B-GA2. Comparison of RMSD values between WT and mutants calculated from 20-ns MD simulations at $300 \mathrm{~K}$ (a). Comparison of normalized RMSF between WT and mutants (b). DCCM analysis of TIGa15B (c) and T/Ga15B-GA2 (d) based on the MD simulation trajectories

was maintained at $4{ }^{\circ} \mathrm{C}$ on a potato dextrose agar plate. Soluble starch, amylose, glycogen, $\alpha$-cyclodextrin, $\beta$-cyclodextrin, and $\gamma$-cyclodextrin were purchased from Sigma-Aldrich. All other chemicals were of analytical grade and commercially available.

\section{Cloning of T/Ga15B and mutants}

T. leycettanus JCM12802 was cultured in the potato dextrose broth (PDB) medium at $40{ }^{\circ} \mathrm{C}$ for 3 days. Total RNA was extracted using the TRIZOL reagent (Invitrogen, Waltham, MA, USA) according to the operation manual. The first strand cDNA was synthesized from the total RNA using the FastKing RT Kit with gDNase (Tiangen, Beijing). Based on the $5^{\prime}$ and $3^{\prime}$-end sequences of the $T l \mathrm{Ga} 15 \mathrm{~B}$ structural gene, the $T l \mathrm{Ga} 15 \mathrm{~B}$-encoding gene devoid of the signal peptide sequence was amplified with gene-specific primers (as shown in Additional file 7). The PCR product was cloned into the plasmid pPIC9 to construct a recombinant plasmid pPIC9-TlGa15B. Site-directed mutants pPIC9-TlGa15B-GA1 and pPIC9$T l \mathrm{Ga} 15 \mathrm{~B}-\mathrm{GA} 2$ were constructed using the Fast Mutagenesis System Kit (TransGen, Beijing) with the plasmid pPIC9-TlGa15B as the template.

\section{Expression and purification of $T / G a 15 B$ and mutants}

The recombinant plasmid PIC9-TlGa15B, pPIC9TlGa15B-GA1, and pPIC9-TlGa15B-GA2 were linearized with BglII and transformed into P. pastoris GS115 competent cells, respectively. The positive transformant with the highest glucoamylase activity was inoculated into YPD medium at $30{ }^{\circ} \mathrm{C}$ for 2 days with shaking at $200 \mathrm{rpm}$ and used as the inoculum of $300 \mathrm{~mL}$ BMGY medium. The cultures were grown at $30{ }^{\circ} \mathrm{C}$ for 2 days, and cells were then harvested and resuspended in $200 \mathrm{~mL}$ BMMY medium for 2 days growth at $30{ }^{\circ} \mathrm{C}$. The crude enzyme was collected by centrifugation at $12,000 \mathrm{~g}$ 


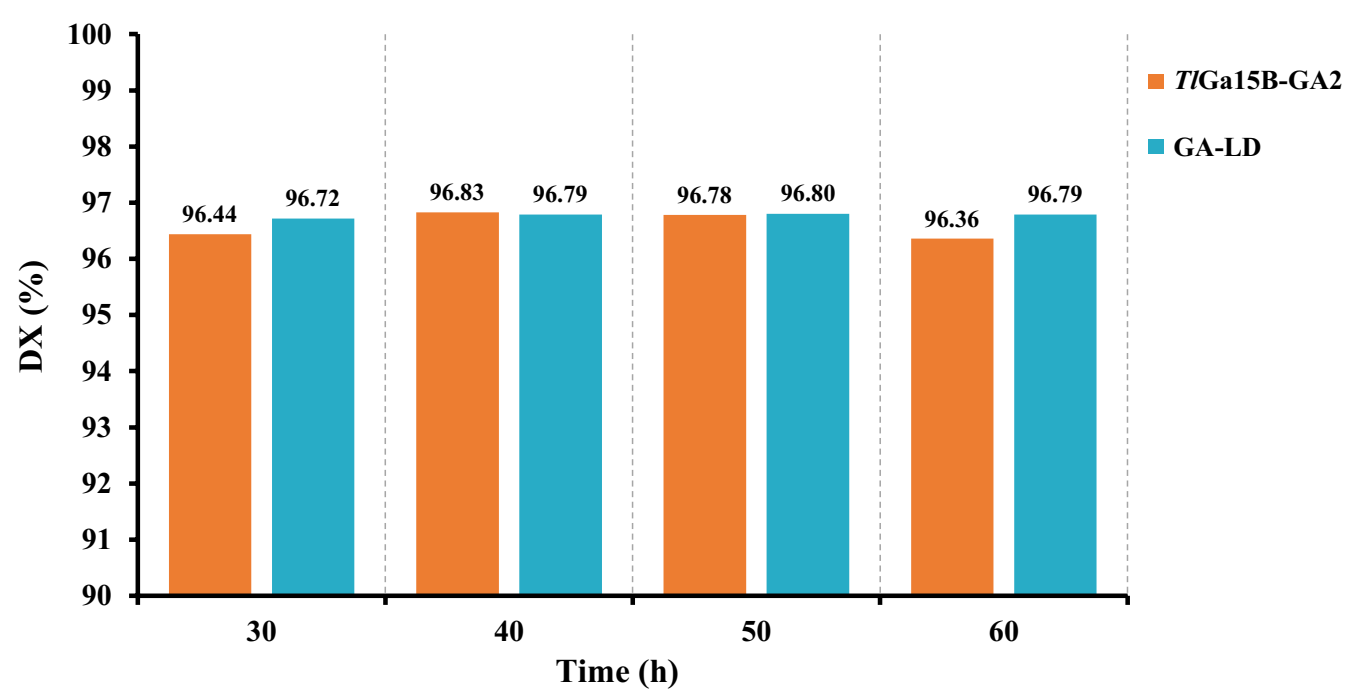

Fig. 5 Comparison of the starch saccharification effect between T/Ga15B-GA2 and the commercial glucoamylase GA-LD in glucose production at $60^{\circ} \mathrm{C}$

for $10 \mathrm{~min}$ at $4{ }^{\circ} \mathrm{C}$, followed by concentration through a $10 \mathrm{kDa}$ cut-off centrifuge filter. The crude enzyme was dialyzed in citrate-phosphate buffer $(20 \mathrm{mM}, \mathrm{pH}$ 6.3) at $4{ }^{\circ} \mathrm{C}$ overnight using a $3 \mathrm{kDa}$ dialysis tube (Vivascience, Hannova, Germany), and run through a HiTrapTM Q Sepharose XL anion exchange column (GE Healthcare, Munich, Germany) for purification. The purified recombinant $T l G a 15 B$ and mutants were verified by SDS-PAGE using a $10 \%$ polyacrylamide gel.

\section{Glucoamylase activity assay}

The glucoamylase activity of $T l \mathrm{Ga} 15 \mathrm{~B}$ and mutants was determined using soluble starch as the substrate. Reactions containing $900 \mu \mathrm{L}$ of $1 \%$ soluble starch in citratephosphate buffer ( $\mathrm{pH} 4.5$ ) and $100 \mu \mathrm{L}$ of appropriately diluted enzyme solution were incubated at $60{ }^{\circ} \mathrm{C}$ for 30 min, using the 3,5-dinitrosalicylic acid (DNS) method to detect the amount of reducing sugar in the reaction. One unit $(\mathrm{U})$ of enzyme activity was defined as the amount of enzyme that released $1 \mu \mathrm{mol}$ of glucose per minute.

\section{Biochemical characterization of purified TIGa15B and mutants}

The effects of $\mathrm{pH}$ on the activity of purified TlGa15B and mutants were determined in glycine- $\mathrm{HCl}(\mathrm{pH} 1-3)$, citric acid- $\mathrm{Na}_{2} \mathrm{HPO}_{4}(\mathrm{pH} 3-8)$, and glycine- $\mathrm{NaOH}(\mathrm{pH}$ 8-12) buffers at $37{ }^{\circ} \mathrm{C}$. To determine $\mathrm{pH}$ stability, the purified $T l G a 15 \mathrm{~B}$ and mutants were incubated in different $\mathrm{pH}(1-12)$ for $1 \mathrm{~h}$ at $37{ }^{\circ} \mathrm{C}$. The effect of temperature on activity was measured in citric acid- $\mathrm{Na}_{2} \mathrm{HPO}_{4}$ buffer $(\mathrm{pH} 4.5)$ at $20-90{ }^{\circ} \mathrm{C}$. To evaluate thermostability, the purified $T l G a 15 B$ and mutants were incubated at $75{ }^{\circ} \mathrm{C}$ for different time periods $(0-60 \mathrm{~min})$.

Kinetic studies were performed in a citric acid$\mathrm{Na}_{2} \mathrm{HPO}_{4}$ buffer ( $\mathrm{pH} 4.5$ ) at $55{ }^{\circ} \mathrm{C}$ using $1.0-10.0 \mathrm{mg} / \mathrm{mL}$ soluble starch as the substrates. The Lineweaver-Burk plot method was used to calculate the $K_{\mathrm{m}}$ and $V_{\max }$ values of the purified $T l \mathrm{Ga} 15 \mathrm{~B}$ and mutants. The substrate specificities of TlGa15B were studied in terms of the hydrolysis of six different substrates, including soluble starch, amylose, glycogen, $\alpha$-cyclodextrin, $\beta$-cyclodextrin, and $\gamma$-cyclodextrin in a citric acid- $\mathrm{Na}_{2} \mathrm{HPO}_{4}$ buffer $(\mathrm{pH} 4.5)$.

\section{Differential scanning calorimetry (DSC) analysis}

The melting temperatures $\left(T_{\mathrm{m}}\right)$ of $T l \mathrm{Ga} 15 \mathrm{~B}$ and mutants were determined using MicroCal $^{\mathrm{TM}}$ VP-Capillary DSC (GE Healthcare). The purified $T l G a 15 B$ and mutants were dissolved in a $10 \mathrm{mM}$ phosphate buffer $(\mathrm{pH} 7.4)$ as an infusion, and the protein concentration was controlled at $0.50 \mathrm{mg} / \mathrm{mL}$. The temperature program was run in the range of $50-100{ }^{\circ} \mathrm{C}$, with a rising rate of $1{ }^{\circ} \mathrm{C} / \mathrm{min}$ and a scanning rate of $1^{\circ} \mathrm{C} / \mathrm{min}$.

\section{Molecular dynamics (MD) simulation and dynamics cross-correlation matrix (DCCM) analysis}

MD simulations were performed using the AMBER14 package at a temperature of $300 \mathrm{~K}$ for $20 \mathrm{~ns}$ with ff99SB force field. The closest distance between periodic box and atom was set $12 \AA$, and the time step was set to 2 fs. Before MD simulation, hydrogen atoms were added and any water molecules that did not interact with the protein were removed, and $20 \mathrm{mM}$ sodium chloride was added to neutralize the charge in the system. The conjugate 
gradient method with $\alpha$-carbon atoms restriction was used for energy minimization. The energy was minimized again without limiting the atoms, and finally the temperature was raised from 0 to $300 \mathrm{~K}$. The MD trajectory data were analyzed using the CPPTRAJ software [36]. In addition, the last $10 \mathrm{~ns}$ trajectories from MD simulations were used to perform DCCM analysis using the Bio3D packages $[37,38]$.

\section{Application of recombinant $T / G a 15 B-G A 2$ in starch saccharification}

To evaluate the industrial application potential of TlGa15B-GA2 in starch saccharification, TlGa15BGA2 was compared with the commercial glucoamylase GA-LD from Shandong Longda Bio-products Co Ltd. The saccharification process of starch was carried out at $60{ }^{\circ} \mathrm{C}$ for 30, 40, 50, and $60 \mathrm{~h}$, containing $50 \mathrm{U} / \mathrm{g} \mathrm{TlGa15B}$ or GA-LD, $0.6 \mathrm{U} / \mathrm{g}$ pullulanase, and $200 \mathrm{~g}$ of liquefied starch. The enzymatic saccharification effect of starch was measured by monitoring the DX value (glucose content) of the saccharification solution.

\section{Abbreviations \\ ETSS: Enzyme thermal stability system; RMSD: Root mean square devia- tion; RMSF: Root mean square fluctuation; PDB: Potato dextrose broth; DNS: 3,5-Dinitrosalicylic acid; MD: Molecular dynamics; DCCM: Dynamics cross- correlation matrix.}

\section{Supplementary Information}

The online version contains supplementary material available at https://doi. org/10.1186/s13068-021-02052-3.

Additional file 1: The Ramachandran plot of homology modeling for T/Ga15B.

Additional file 2: SDS-PAGE analysis of the recombinant T/Ga15B and mutants. Lane 1, 3, 5, the culture supernatant of transformants T/Ga15B, T/Ga15-GA1, and T/Ga15-GA2; lane 2, 4, 6, the purified T/Ga15B, T/Ga15GA1, and TIGa15-GA2.

Additional file 3: The thermostability of the purified recombinant T/Ga15B.

Additional file 4 LC-MS/MS analysis of the formation of disulfide bonds in the T/Ga15B mutants. a: the disulfide-linked peptide SNPSGGLCT/ SASGPCA (132C/492C); b: the disulfide-linked peptide PLWYCIV/SAIPCSA $(548 \mathrm{C} / 562 \mathrm{C})$

Additional file 5: The modeled structure of T/Ga15B-GA2. Six candidate mutated sites away from the catalytic center were indicated in blue balls. Two disulfide bonds were indicated as yellow sticks.

Additional file 6: The specific activity of $T / G a 15 B$ and its mutants in the temperature range from 20 to $90{ }^{\circ} \mathrm{C}$.

Additional file 7: Primers used in this study.

\section{Acknowledgements}

Not applicable.

\section{Authors' contributions}

BY and HYL conceived and designed the experiments. LGT, JZ, and XW performed the experiments. XLW, HQH, HMY, and TT analyzed the data. YW and
YGB contributed analytic tools. LGT and XQ wrote the manuscript. HYL and XQ reviewed and revised the manuscript. All the authors read and approved the final manuscript.

\section{Funding}

This research was supported by the National Key Research and Development Program of China (2021YFC2100400), the State Key Laboratory of Animal Nutrition Project (2004DA125184G2101), and the China Agriculture Research System of MOF and MARA (CARS-41).

\section{Availability of data and materials}

The datasets used and/or analyzed during the current study are available from the corresponding author on reasonable request.

\section{Declarations}

Ethics approval and consent to participate

Not applicable.

\section{Consent for publication \\ Not applicable.}

\section{Competing interests}

The authors declare that they have no competing interests.

Received: 10 August 2021 Accepted: 2 October 2021

Published online: 16 October 2021

\section{References}

1. Kumar P, Satyanarayana T. Microbial glucoamylases: characteristics and applications. Crit Rev Biotechnol. 2009;29:225.

2. Sauer J, Sigurskjold BW, Christensen U, Frandsen TP, Mirgorodskaya E, Harrison M, Roepstorff P, Svensson B. Glucoamylase: structure/function relationships, and protein engineering. Biochim et Biophys Acta (BBA) Protein Struct Mol Enzymol. 2000;1543:275-93.

3. Wang C, Yang L, Luo L, Tang S, Wang Q. Purification and characterization of glucoamylase of Aspergillus oryzae from Luzhou-flavour Daqu. Biotech Lett. 2020:42:2345-55.

4. Carrasco M, Alcaíno J, Cifuentes V, Baeza M. Purification and characterization of a novel cold adapted fungal glucoamylase. Microb Cell Fact. 2017; $16: 75$

5. Parashar D, Satyanarayana T: An insight into ameliorating production, catalytic efficiency, thermostability and starch saccharification of acidstable a-amylases from acidophiles. Front Bioeng Biotechnol. 2018;6.

6. Lago MC, dos Santos FC, Bueno PSA, de Oliveira MAS, Barbosa-Tessmann IP. The glucoamylase from Aspergillus wentii: purification and characterization. J Basic Microbiol. 2021;61:443-58.

7. Karim KMR, Husaini A, Sing NN, Tasnim T, Mohd Sinang F, Hussain H, Hossain MA, Roslan H. Characterization and expression in Pichia pastoris of a raw starch degrading glucoamylase (GA2) derived from Aspergillus flavus NSH9. Protein Exp Purif. 2019:164:105462.

8. Tanaka Y, Konno N, Suzuki T, Habu N. Starch-degrading enzymes from the brown-rot fungus Fomitopsis palustris. Protein Exp Purif. 2020;170:105609.

9. Zheng Y, Xue Y, Zhang Y, Zhou C, Schwaneberg U, Ma Y. Cloning, expression, and characterization of a thermostable glucoamylase from Thermoanaerobacter tengcongensis MB4. Appl Microbiol Biotechnol. 2010:87:225-33.

10. He Z, Zhang L, Mao Y, Gu J, Pan Q, Zhou S, Gao B, Wei D. Cloning of a novel thermostable glucoamylase from thermophilic fungus Rhizomucor pusillus and high-level co-expression with a-amylase in Pichia pastoris. BMC Biotechnol. 2014;14:114.

11. Chen J, Zhang YQ, Zhao CQ, Li AN, Zhou QX, Li DC. Cloning of a gene encoding thermostable glucoamylase from Chaetomium thermophilum and its expression in Pichia pastoris. J Appl Microbiol. 2007;103:2277-84.

12. Kim M, Park J, Kim Y, Lee H, Nyawira R, Shin H, Park C, Yoo S, Kim Y, Moon T, Park K. Properties of a novel thermostable glucoamylase from the hyperthermophilic archaeon Sulfolobus solfataricus in relation to starch processing. Appl Environ Microbiol. 2004;70:3933. 
13. Schmidt A, Shvetsov A, Soboleva E, Kil Y, Sergeev V, Surzhik M. Thermostability improvement of Aspergillus awamori glucoamylase via directed evolution of its gene located on episomal expression vector in Pichia pastoris cells. Protein Eng Des Sel. 2019;32:251-9.

14. Liu H, Wang W. Protein engineering to improve the thermostability of glucoamylase from Aspergillus awamori based on molecular dynamics simulations. Protein Eng Des Sel. 2003:16:19-25.

15. McDaniel A, Fuchs E, Liu Y, Ford C. Directed evolution of Aspergillus niger glucoamylase to increase thermostability. Microb Biotechnol. 2008;1:523-31.

16. Wang Y, Fuchs E, Silva RD, Mc Da Niel A, Seibel J, Ford C. Improvement of Aspergillus niger glucoamylase thermostability by directed evolution. Starch Strike. 2010;58:501-8.

17. Kurahashi R, Tanaka S, Takano K. Activity-stability trade-off in random mutant proteins. J Biosci Bioeng. 2019;128:405-9.

18. Siddiqui KS. Defying the activity-stability trade-off in enzymes: taking advantage of entropy to enhance activity and thermostability. Crit Rev Biotechnol. 2017;37:309-22.

19. Contreras F, Thiele MJ, Pramanik S, Rozhkova AM, Schwaneberg U. KnowVolution of a GH5 cellulase from Penicillium verruculosum to improve thermal stability for biomass degradation. ACS Sustain Chem Eng. 2020;8:12388-99.

20. Shivange AV, Roccatano D, Schwaneberg U. Iterative key-residues interrogation of a phytase with thermostability increasing substitutions identified in directed evolution. Appl Microbiol Biotechnol. 2016;100:227-42.

21. You S, Tu T, Zhang L, Wang Y, Huang H, Ma R, Shi P, Bai Y, Su X, Lin Z, et al. Improvement of the thermostability and catalytic efficiency of a highly active $\beta$-glucanase from Talaromyces leycettanus JCM12802 by optimizing residual charge-charge interactions. Biotechnol Biofuels. 2016;9:124.

22. Wang X, Ma R, Xie X, Liu W, Tu T, Zheng F, You S, Ge J, Xie H, Yao B, Luo H. Thermostability improvement of a Talaromyces leycettanus xylanase by rational protein engineering. Sci Rep. 2017;7:15287.

23. Gu Y, Zheng F, Wang Y, Su X, Bai Y, Yao B, Huang H, Luo H. Characterization of two thermophilic cellulases from Talaromyces leycettanus JCM12802 and their synergistic action on cellulose hydrolysis. PLOS ONE. 2019;14:e0224803-e0224803.

24. Li X, Xia W, Bai Y, Ma R, Yang H, Luo H, Shi P. A novel thermostable GH3 $\beta$-glucosidase from Talaromyce leycettanus with broad substrate specificity and significant soybean isoflavone glycosides-hydrolyzing capability. Biomed Res Int. 2018;2018:4794690.

25. Wang C, Luo H, Niu C, Shi P, Huang H, Meng K, Bai Y, Wang K, Hua H, Yao B. Biochemical characterization of a thermophilic $\beta$-mannanase from Talaromyces leycettanus JCM12802 with high specific activity. Appl Microbio Biotechnol. 2015;99:1217-28.

26. Almagro Armenteros JJ, Tsirigos KD, Sønderby CK, Petersen TN, Winther O, Brunak S, von Heijne G, Nielsen H. SignalP 5.0 improves signal peptide predictions using deep neural networks. Nat Biotechnol. 2019;37:420-3.
27. Nielsen BR, Lehmbeck J, Frandsen TP. Cloning, heterologous expression, and enzymatic characterization of a thermostable glucoamylase from Talaromyces emersonii. Protein Expr Purif. 2002;26:1-8.

28. Lee J, Paetzel M. Structure of the catalytic domain of glucoamylase from Aspergillus niger. Acta Crystallogr Sect F Struct Biol Cryst Commun. 2011;67:188-92.

29. MacauleyPatrick S, Fazenda ML, McNeil B, Harvey LM. Heterologous protein production using the Pichia pastoris expression system. Yeast. 2005:22:249-70.

30. Xian L, Feng J. Purification and biochemical characterization of a novel mesophilic glucoamylase from Aspergillus tritici WZ99. Int J Biol Macromol. 2018;107:1122-30.

31. Hua H, Luo H, Bai Y, Wang K, Niu C, Huang H, Shi P, Wang C, Yang P, Yao B. A thermostable glucoamylase from Bispora sp. MEY-1 with stability over a broad $\mathrm{pH}$ range and significant starch hydrolysis capacity. PLOS ONE. 2014;9:e113581.

32. Karim KMR, Husaini A, Hossain MA, Sing NN, Mohd Sinang F, Hussain MHM, Roslan HA. Heterologous, expression, and characterization of thermostable glucoamylase derived from Aspergillus flavus NSH9 in Pichia pastoris. Biomed Res Int. 2016;2016:5962028-5962028.

33. Zhu B, Wang D, Wei N. Enzyme discovery and engineering for sustainable plastic recycling. Trends Biotechnol. 2021.

34. Zhang L, Tang X, Cui D, Yao Z, Gao B, Jiang S, Yin B, Yuan YA, Wei D. A method to rationally increase protein stability based on the chargecharge interaction, with application to lipase LipK107. Protein Sci. 2014;23:110-6.

35. Wang R, Wang $S, X u Y, Y u X$. Enhancing the thermostability of Rhizopus chinensis lipase by rational design and MD simulations. Int J Biol Macromol. 2020;160:1189-200.

36. Roe DR, Cheatham TE. PTRAJ and CPPTRAJ: software for processing and analysis of molecular dynamics trajectory data. J Chem Theory Comput. 2013;9:3084-95.

37. Skjærven L, Yao X, Scarabelli G, Grant BJ. Integrating protein structural dynamics and evolutionary analysis with Bio3D. BMC Bioinformat. 2014;15:399.

38. Grant BJ, Rodrigues APC, ElSawy KM, McCammon JA, Caves LSD. Bio3d: an $R$ package for the comparative analysis of protein structures. Bioinformatics. 2006:22:2695-6.

\section{Publisher's Note}

Springer Nature remains neutral with regard to jurisdictional claims in published maps and institutional affiliations.
Ready to submit your research? Choose BMC and benefit from:

- fast, convenient online submission

- thorough peer review by experienced researchers in your field

- rapid publication on acceptance

- support for research data, including large and complex data types

- gold Open Access which fosters wider collaboration and increased citations

- maximum visibility for your research: over 100M website views per year

At BMC, research is always in progress.

Learn more biomedcentral.com/submissions 